\title{
Effect of glass pre-treatment on the nucleation of semi-transparent gold coatings
}

\author{
Xiaoda $\mathrm{Xu}^{\mathrm{a}, \mathrm{b}}$, Michael B. Cortie, ${ }^{\mathrm{a}^{*}}$ and Michael Stevens ${ }^{\mathrm{b}}$ \\ a Institute for Nanoscale Technology, University of Technology, Sydney NSW 2007, \\ Australia. \\ ${ }^{b}$ Department of Chemistry, Materials and Forensics, University of Technology \\ Sydney, NSW 2007, Australia.
}

Corresponding author: Tel: 612 95142208; Fax:61 295147553

E-mail: Michael.Cortie@uts.edu.au

\section{Abstract}

Coatings of gold nanoparticles with a uniform film texture and a neutral blue hue may be applied to glass by an aqueous process, and such coatings have recently been proposed for architectural applications. Here we show that the optical transmission spectrum of these coatings is directly related to the interplay between the nucleation, growth and aggregation of the particles. In particular, prior treatments of the glass substrate in 1:1 sulfuric acid $\left(\mathrm{H}_{2} \mathrm{SO}_{4}\right), 98 \% \mathrm{H}_{2} \mathrm{SO}_{4}$ and buffered hydrofluoric acid (HF) exerted a strong influence on the subsequent particle size and coverage, which is explained here in terms of contact angle and its effect on rates of nucleation. The rate of nucleation on the surface treated with HF is estimated to be twice that of the surface treated with $98 \% \mathrm{H}_{2} \mathrm{SO}_{4}$. The color of the coatings is the result of interparticle plasmonic interactions, and is therefore also controlled by the nature of nucleation and growth process. 
Keywords: nanostructures, nucleation, optical properties 


\section{Introduction}

Ordinary window glass is almost transparent in both the visible and the nearinfrared [1], and, in summer, this leads to the transmission into a building of both the desired visible and the undesired infrared wavelengths. Approximately $51 \%$ of the power of typical solar radiation is in the infrared [2], and glazing systems that can selectively attenuate this have wide spread applications. The use of vacuum-deposited gold coatings on glass for this purpose is now well-known, although nowadays there are several other options available [3-5]. In hot climates or seasons, the use of these coatings can substantially reduce energy consumption arising from air conditioning. Unfortunately, all of the coatings applied by vacuum deposition are expensive, due primarily to the high cost of the initial investment in manufacturing equipment, and the relatively long times required for the deposition. In contrast, the cost of the raw materials in the coatings is modest, even if gold is used [5].

We have previously argued that the deposition of gold nanoparticles by a low temperature, wet chemical process has significant possibilities for this application [5], since this is potentially far cheaper than vacuum sputtering or chemical vapor deposition. Thin films with a neutral blue color and useful solar screening capabilities have been produced $[5,6]$, and the hue seems to be generally acceptable to the potential end-users. The process is quite flexible, and could, in principle, be applied on the tail end of an existing float glass production line, or off-line. The coating material is inert and, provided that it is not abraded, should survive for the lifetime of the window. However, there are still some disadvantages associated with these coatings. In particular, they exhibit only a modest degree of spectral selectivity 
between the visible and near infrared region [7], with a figure-of-merit, $T_{\mathrm{vis}} / T_{\mathrm{sol}}$, of $\sim 0.8$, where $T_{\text {vis }}$ is the proportion of the visible spectrum that is transmitted and $T_{\text {sol }}$ the proportion of the total solar spectrum transmitted [6]. Ideally, a solar control application requires $100 \%$ blocking of the infra-red, and $100 \%$ transmission of the visible, corresponding to a $T_{\mathrm{vis}} / T_{\text {sol }}$ of 2.08 for standard solar radiation [6]. In addition, it would be useful if a wider range of color tones and greater control of them was available in these coatings.

Here we show that the transmission spectra of the gold nanoparticle coatings is directly related to the nature of their nucleation and growth on the glass surface. This can cause coatings of similar gold content, but different patterns of aggregation, to exhibit quite different transmission spectra. These effects may be controlled by addressing the nature of the glass surface (which largely controls the rate of nucleation of particles) or by manipulation of parameters such as the $\mathrm{pH}$ or temperature of the deposition solution (which tend to control the rate of growth).

\section{Experimental}

Ethylene diamine, hydrazine, and sodium hydroxide were obtained from Aldrich, while $\mathrm{HCl}, \mathrm{HNO}_{3}$ were from $\mathrm{BDH}, \mathrm{H}_{2} \mathrm{SO}_{4}$, was obtained from Q-Store, $\mathrm{Na}_{2} \mathrm{~B}_{4} \mathrm{O}_{7} \cdot 10 \mathrm{H}_{2} \mathrm{O}$ was purchased from Unilab, $\mathrm{SnCl}_{2} \cdot 6 \mathrm{H}_{2} \mathrm{O}$ and $\mathrm{HF}$ were from Sigma, $\mathrm{NH}_{4} \mathrm{HF}_{2}$ was from AJAX Univar. Gold was sourced from AGR-Matthey. All chemicals were used as received. All $\mathrm{H}_{2} \mathrm{O}$ used was purified by double-distillation. $0.10 \mathrm{M} \mathrm{HAuCl}_{4}$ solution was prepared by dissolving pure gold in aqua regia $(\mathrm{HCl}$ : 
$\left.\mathrm{HNO}_{3} 3: 1 \mathrm{~V} / \mathrm{V}\right)$. The method may be found elsewhere [5]. It is accepted that some residual $\mathrm{HCl}$ content may still present in the solution. Buffered hydrofluoric acid (HF) was prepared by dissolving $20 \mathrm{~g} \mathrm{NH}_{4} \mathrm{HF}_{2}, 5 \mathrm{ml} 49 \% \mathrm{HF}$ and $100 \mathrm{ml}$ of $37 \% \mathrm{HCl}$ in 1 L distilled water.

Generic soda-lime glass microscope slides (1 $\mathrm{mm}$ thick) were obtained from Livingstone, and their surfaces were pre-processed in one of three ways, Table 1. These treatments were selected to provide different kinds of surface, $a$, because it is well known to oxidize organic contaminants, $b$, because it is known to both produce a nanoscale rough surface on glass and remove sodium [8] and $c$ because it reputedly etches the glass to a smooth finish (it is an industry standard solution applied to pretreat glass prior to coating[9], [10].) Thereafter, the slides were pretreated for 2 minutes in $0.1 \%$ aqueous $\mathrm{SnCl}_{2}$, rinsed with pure water, and exposed to the coating solution.

The coating solution must be made up immediately before use, and comprised $0.0004 \mathrm{M} \mathrm{HAuCl}_{4}, 0.015 \mathrm{M} \mathrm{N}_{2} \mathrm{H}_{4}$ and $0.03 \mathrm{M}$ ethylene diamine in $\mathrm{pH} 8$ buffer which was made with $100 \mathrm{ml}$ of $0.025 \mathrm{M} \mathrm{Na}_{2} \mathrm{~B}_{4} \mathrm{O}_{7} \cdot 10 \mathrm{H}_{2} \mathrm{O}$ (borax) and $41 \mathrm{ml}$ of $0.1 \mathrm{M} \mathrm{HCl}$. It was confirmed that the $\mathrm{pH}$ stayed constant during the deposition. Deposition on the HF-treated surface ( $\operatorname{surface} c$ ) was much faster than for the other two.

A Cary 5E UV/Vis/IR spectrophotometer, working in the range of $170 \mathrm{~nm}$ to 4300 $\mathrm{nm}$ and with a resolution of $0.5 \mathrm{~nm}$ was used to inspect the visible-IR transmission spectrum of the samples. The scanning rate was $5 \mathrm{~nm} / \mathrm{s}$ with spectral bandwidth (SBW) of $2 \mathrm{~nm}$. X-ray photoelectron spectroscopy (XPS) spectra of the surface of the 
glass samples were recorded using $\mathrm{Al} \mathrm{K}_{\alpha}$ radiation $(1486.6 \mathrm{eV})$ at $20 \mathrm{eV}$ and $10^{-9}$ Torr. The experimental data were calibrated with the carbon peak that was always shown as a contamination at $285 \mathrm{eV}$. The dynamic contact angles (DCA) were obtained with a Cahn DCA-322 DCA analyzer using water. A stage speed of $200 \mathrm{~mm} / \mathrm{s}$ was applied to advance and recede the sample from the liquid. The surface energies reported are the average of five measurements.

The morphology of deposited gold was characterized using a LEO scanning electron microscope (SEM) with in-lense images at 2-30 kV, and with a Dimension 3100 atomic force microscope (AFM). The distribution of gold nanoparticle diameters at different stages of the deposition was obtained from the SEM images using customwritten software. The software allowed the user to map an image of the coating into an array of hemispheres, which could then be analyzed further. The height-todiameter ratio of the particles was obtained from the AFM image files using WSxM 3.0 of Nanotec Electronica, Spain. Seventy particles were sampled on each surface. The radii measured on the AFM were corrected with Equation (1), which is determined from SEM images of the same samples and a hardball model of AFM tips [11], and accounts for the fact that the AFM over-estimates such distances due to interference of the tip with the sides of the nanoparticle (Fig.1)

$$
r_{P}=\sqrt{R_{t}^{2}+r_{O b s}^{2}}-R_{t}
$$

In Equation (1), $r_{P}$ is the radius of real gold nanoparticle, $R_{t}$ is the radius of the AFM tip, $r_{\text {obs }}$ is the radius of gold nanoparticle observed with AFM. $R_{t}$ was around 10 $\mathrm{nm}$ according to the manufacturer's (Ultrasharp $\left.{ }^{\circledR}\right)$ information. 


\section{Results}

The XPS spectra of the glass surfaces after different pretreatments are shown in Fig.

2. No sodium was found on the surface after etching with $\mathrm{H}_{2} \mathrm{SO}_{4}$, despite this element being a significant constituent of the type of glass used. On the other hand, there was still large amount of sodium remaining on the surface after application of the buffered HF. The content of silicon remained the same with the different treatments.

The average DCA results for $98 \% \mathrm{H}_{2} \mathrm{SO}_{4}, 1: 1 \mathrm{H}_{2} \mathrm{SO}_{4}$ and buffered $\mathrm{HF}$ are 0.044, 0.072 and $0.071 \mathrm{~J} / \mathrm{m}^{2}$ respectively. After etching with $98 \% \mathrm{H}_{2} \mathrm{SO}_{4}$, the surface of glass was hydrophobic rather than hydrophilic and its surface tension is markedly lower than those of the surfaces etched with 1:1 $\mathrm{H}_{2} \mathrm{SO}_{4}$ or buffered HF. The surface tensions of the latter two were almost the same. The morphologies of the etched glass surfaces with different pretreatments are shown in Fig. 3. Even though the RMS (root mean square ) roughness for the glass surfaces treated with $98 \% \mathrm{H}_{2} \mathrm{SO}_{4}$ and buffered $\mathrm{HF}$ are similar, (being $1.94 \mathrm{~nm}$ and $2.06 \mathrm{~nm}$ the textures of surfaces are quite different. The morphology of the glass surface etched with HF displays an irregular lamellar structure, while that of the surface etched with $\mathrm{H}_{2} \mathrm{SO}_{4}$ shows mounds.

The optical transmission spectra of gold coatings deposited on the different glass surfaces are indicated in Fig. 4. It was found that gold particles grown on the surfaces treated with $\mathrm{H}_{2} \mathrm{SO}_{4}$ tended to develop dual absorption peaks at around $520 \mathrm{~nm}$ and $700 \mathrm{~nm}$. However, coatings deposited on the glass surface treated with buffered HF manifested a single absorption peak at about $700 \mathrm{~nm}$. 
The morphologies of the gold coatings on the different glass surfaces are shown in Fig. 5, with the samples shown corresponding to the spectra in Fig. 4. It is evident that the texture of the gold coating on the surface etched with HF is more uniform than that etched with $\mathrm{H}_{2} \mathrm{SO}_{4}$. Analysis of the SEM images showed that the distribution of particle size is broader on the surface etched with $98 \% \mathrm{H}_{2} \mathrm{SO}_{4}$ (Fig. 6). Some diameters were more than $100 \mathrm{~nm}$ (Fig. 6a), while others were around $40 \mathrm{~nm}$. On the surface etched with buffered HF, the particles were more uniform and around $50 \mathrm{~nm}$ in diameter (Fig. 6 c). There are also more isolated gold nanoparticles in the surface etched with $\mathrm{H}_{2} \mathrm{SO}_{4}$ (Fig. 5 a, b), while most of the gold particles on the surface etched with buffered HF are within close proximity to one another.

The AFM images of gold nanoparticles in the initial stages are shown in Fig. 7. Similar to the SEM images shown in Fig. 5, the gold nanoparticles that nucleated on the surface pretreated with buffered HF are smaller and more uniform. The shape of the particles is further explored in Fig. 8, which shows the ratio of radius $(R)$ to height $(H)$ of the particles deposited on the surfaces etched with $98 \% \mathrm{H}_{2} \mathrm{SO}_{4}$ and buffered HF, as measured using an AFM (Fig. 7). These particular samples were treated in the deposition solution for a shorter length of time than the ones described in the preceding paragraph in order that the particles would be smaller and sufficiently well separated for measurement of their dimensions. The mean value of $R / H$ for the $\mathrm{H}_{2} \mathrm{SO}_{4}$-treated surface is $3.43(s=1.20)$ whereas that of the HF-treated surface was $4.92(s=0.99)$. Obviously, the particles deposited on the surface pre-treated with HF are on average considerably flatter than those formed on the surface pre-treated with $98 \% \mathrm{H}_{2} \mathrm{SO}_{4}$. 


\section{Discussion}

\subsection{Effect of surface energy on nucleation of gold nanoparticles}

The morphology, and hence the color (e.g. $[5],[12,13])$, of the coatings is largely controlled by the rate at which new particles nucleate on the surface, and their subsequent rate of growth. Classic nucleation theory $[14,15]$ is based on the premise that random aggregations of atoms occur spontaneously to form nuclei of radius $r$. If $W_{\mathrm{f}}$ is the work required to form a nucleus, then any randomly-formed nucleus can only grow if $\mathrm{d} W_{\mathrm{f}} / \mathrm{d} r$ is $<0$. The theory predicts that there is a critical size, $r^{*}$, and volume, $V^{*}$, of randomly-formed nucleus, above which $\mathrm{d} W_{\mathrm{f}} / \mathrm{d} r<0$, and below which re-dissolution will occur. $W_{\mathrm{f}}$ may be estimated from a consideration of the interplay of the driving force of the chemical reaction or phase transformation with the surface energies $\gamma_{\mathrm{Au} / \mathrm{H} 20}$ and $\gamma_{\mathrm{Au} / \mathrm{glass}}$ required to create the nucleus. In the present case the problem may be somewhat simplified by assuming a time-independent driving force, $F$ of $\sim-200 \mathrm{~kJ}$ per mole of $\mathrm{Au}$, or $\sim-2 \times 10^{10} \mathrm{~J}$ per $\mathrm{m}^{3}$ of $\mathrm{Au}^{0}$ deposited, derived from the $\Delta \mathrm{G}$ of the reduction reaction

$$
2 \mathrm{HAuCl}_{4}+2 \mathrm{~N}_{2} \mathrm{H}_{4} \rightarrow 2 \mathrm{Au}+8 \mathrm{HCl}+2 \mathrm{~N}_{2}+\mathrm{H}_{2}
$$

and the assumption of a cap-shaped particle of gold on the glass surface. $F$ is independent of time since, in this work at least, only the very early portion of gold deposited from the solution is taken up onto the test specimens, with the remainder being wasted by precipitation later on after the test samples have already been removed from the bath. The interfacial free energy, $\gamma_{\mathrm{Au} / \mathrm{H} 20}$ of gold is likely to be of the order of $1 \mathrm{~J} / \mathrm{m}^{2}$, since that of a molten gold surface exposed to an insert environment is in the range 0.75 to $1.1 \mathrm{~J} / \mathrm{m}^{2}$ [16]. Generally, certain other algebraic and geometric 
simplifications are also applied to this problem, and, since the driving force here is assumed to be a constant, the work to create such a particle may be expressed as [15]

$$
W_{f}=\left\{1-\frac{3}{2} \cos \theta+\frac{1}{2} \cos ^{3} \theta\right\}\left\{2 \pi r^{2} \gamma_{A u / H 2 O}-\frac{2 \pi r^{3}}{3} F\right\}
$$

where $\theta$ in this case is the contact angle of the gold nucleus on the glass. Theta may be derived from the shape of the particle (Fig. 8) using the expression

$$
\theta=90-\arccos \left[\frac{2 R H}{R^{2}+H^{2}}\right]
$$

Mean values for $\theta$ of $43.4^{\circ}\left(\mathrm{s}=11.8^{\circ}\right)$ and $29.7^{\circ}\left(\mathrm{s}=6.6^{\circ}\right)$ were obtained for the surfaces treated with $98 \% \mathrm{H}_{2} \mathrm{SO}_{4}$ and buffered $\mathrm{HF}$ respectively. It is obvious that the net effect of pre-treating the glass with buffered HF has been to lower $\gamma_{\mathrm{Au} / \mathrm{glass}}$ and thereby lower $\theta$.

When $\mathrm{dWf} / \mathrm{dr}=0$ in (3), the resulting nucleus radius and volume are termed $r^{*}$ and $\mathrm{V}^{*}$, the critical radius and volume, since any further growth will result in $\mathrm{d} W / \mathrm{d} r$ becoming negative, and therefore spontaneous. Surprisingly, the algebra shown in [15] that the value of $r^{*}$ does not depend upon $\theta$ at all, however, the critical volume of nucleus derived from (5) obviously does:

$$
V^{*}=\frac{16 \pi}{3}\left(\frac{\gamma_{A u / H 20}}{F}\right)^{3}\left(1-\frac{3}{2} \cos \theta+\frac{1}{2} \cos ^{3} \theta\right)
$$


$V^{*}$ for the HF-treated surface $\left(\theta \approx 30^{\circ}\right)$ is reduced to only $24 \%$ that of $V^{*}$ of the surface treated with the $\mathrm{H}_{2} \mathrm{SO}_{4}\left(\theta \approx 43^{\circ}\right)$. Since a random aggregation of atoms must occur to achieve $V^{*}$, and the rate at which this event occurs at a given temperature is given approximately by

$$
I \propto \exp ^{-V^{*}}
$$

it follows that formation of critically-sized nuclei will proceed at about twice the rate on the HF-treated surface.

Accurate extraction of $r^{*}, V^{*}$ or $I$ from these data requires more analysis than we can present here. However, we note that the effect described will facilitate and accelerate the nucleation process on the HF-treated surface, causing a coating of more uniform particle size to form on it. Conversely, a slower rate of nucleation generally produces a broader distribution of particle sizes, with a less regular coating morphology because particles formed earlier on have more time to grow. These trends may be discerned in the histograms of Fig. 6. Finally we note that from (5) and (6) that since $F$ is very large in this system and $\gamma_{\mathrm{Au} / \mathrm{H} 20}$ is comparatively far smaller, $V^{*}$ will always be very small, possibly less than $2 \mathrm{~nm}$, and $I$ will always be very large.

Heterogeneous nucleation, although prevalent in the gold deposition process, is quite difficult to model because of a lack of knowledge of the interactions between the gold nanoparticles and the activated glass. Nevertheless, the Gibbs free energy of the system is a reasonable indicator of the thermodynamic stability of different sizes of gold nano-clusters. This may be estimated as follows:

For a spherical cap with $n$ gold atoms, the reversible work of formation is given by: 


$$
\Delta G_{n}=n \Delta G^{\prime}+\sum A_{i} \gamma_{i}
$$

)

where where $\Delta \mathrm{G}^{\prime}$ is the Gibbs free energy per atom of the new phase, less that of the initial phase, $A_{\mathrm{i}}$ is the surface area of a cluster of $n$ atoms, and $\gamma_{\mathrm{i}}$ is the interfacial free energy per unit area

According to classic nucleation theory, the interfacial energy between substrate and liquid (SL), crystal and substrate (CS), crystal and liquid (CL) have the balance:

$$
\gamma_{S L}=\gamma_{C S}+\gamma_{C L} \cdot \cos \theta
$$

in which $\theta$ is the contact angle measured between the substrate and the nucleus cap. Using the geometrical relationship of this system, it can be shown that

$$
\Delta G_{n}=n \Delta G^{\prime}+\frac{f(\theta) \sigma_{C L}}{\beta \cdot r} \cdot 2 n \pi r_{A u}^{3}
$$

where $\beta$ is the packing density of face centred (FCC) lattice $(0.74)$ and

$$
f(\theta)=\frac{(2+\cos \theta)(1-\cos \theta)^{2} \cdot \sin \theta}{1-\frac{3}{2} \cos \theta+\frac{1}{2} \cos ^{3} \theta}
$$

The Gibbs free energy of $10^{-4}$ mol of gold atoms deposited as uniform hemispherical caps on surfaces of different interfacial free energy surface is shown in Fig. 9. From this simulation, it is evident that smaller clusters are stable on the surface etched with buffered HF.

These results strongly suggest that interlayers or pretreatments that can promote the wetting ability of glass with respect to gold may be essential in the production of transparent gold nanocoatings on glass. 


\section{2 Color and IR reflectance available from gold nanoparticle coatings}

The plasmon resonance peak of isolated gold nanoparticles occurs in water at around $520 \mathrm{~nm}$ and has been exhaustively studied in the past. Unfortunately, it is only susceptible to rather modest variations in position. For example, it may be red shifted a few tens of nanometers by an increase of the particle diameter or of the dielectric constant of the ambient medium, but these small changes have little significance in the context of the present application. Rather, most of the useful functionality in the present system comes from absorption peak at $\sim 700 \mathrm{~nm}$. 
The peak at $\sim 700 \mathrm{~nm}$ is clearly due to dipole-dipole interactions between particles that are close together [17-23]. However, a question arises in respect of the interpretation of the first peak in the present spectra. Obviously, the presence of this peak in the spectrum of an ensemble of gold nanoparticle aggregates can in many instances arise from a straight forward superposition of the spectra of isolated particles and aggregated particles e.g. [19]. However, does a peak in this position always prove the presence of isolated particles? Could, it have arisen, for example from a quadrupole resonance in the present particles, which are hemispherical, or from some other source? In support of the first, and simpler, possibility, we note that the $520 \mathrm{~nm}$ peak is absent from the spectra of uniform aggregations of monodisperse particles e.g. [20, 21], but has been observed in the spectra of random aggregates of spherical particles $[19,22,23]$. The next issue is whether the particles on those of our surfaces treated with sulphuric acid (Figure 5) are sufficiently isolated to support this explanation. Red-shifting of the plasmon resonance is acutely sensitive to the ratio of particle radius $(r)$ to centre-to-centre distance $(D)$ between particles. Published data shows that a plasmon resonance in the range of 520 to $550 \mathrm{~nm}$ is maintained as long as $r / D$ less than about $0.4[24,25](r / D=0.5$ for two particles that touch). It is therefore clear, for example, that the surface-to-surface distance between two particles of $30 \mathrm{~nm}$ diameter must be less than $7 \mathrm{~nm}$ for the absorption peak to be red shifted beyond 550 $\mathrm{nm}$. The presence of the $520 \mathrm{~nm}$ peak in the spectra of coatings on the surfaces treated with sulphuric acid is evidence that many of the particles are effectively more than 7 $\mathrm{nm}$ distant from their neighbors.

For the glass surface treated with sulfuric acid, the two peaks mentioned above developed concurrently. First, the isolated particles grew and second, some of the 
particles aggregated as clusters. Therefore two plasmon resonances are evident in the spectrum. However, for the glass surface treated with buffered HF, nucleation happened so fast that even at the very beginning stage the density of gold particles was high. There was therefore no space for large, isolated particles to develop, and a large number of smaller particles agglomerated from an early stage during the deposition. These factors also restricted the growth of the nanoparticles themselves. The resulting plasmon resonance at $720 \mathrm{~nm}$ was so strong that it obscured any residual signal at $520 \mathrm{~nm}$.

The surface tension, $\gamma_{\mathrm{glass} / \mathrm{H} 20}$ of the surface etched with $1: 1 \mathrm{H}_{2} \mathrm{SO}_{4}$ is quite similar to that etched with buffered HF. However, its optical spectrum and particle morphology was like that of the surface treated with $98 \% \mathrm{H}_{2} \mathrm{SO}_{4}$. The gold nanoparticle coating on this surface contained both agglomerated and isolated particles, and had the associated dual plasmon absorptions at $520 \mathrm{~nm}$ and $700 \mathrm{~nm}$. The reason, we believe, is that $\gamma_{\mathrm{Au} / \mathrm{glass}}$ is the more important factor controlling particle shape in this system, and it was evidently similar in magnitude for both of the surfaces treated with $\mathrm{H}_{2} \mathrm{SO}_{4}$.

The nature of the formation of gold nuclei and their growth is very important in these coatings. It is evident from their morphology that the nucleation process of chemically-deposited gold on glass surfaces follows the so-called Volmer-Weber mode in so far as separate islands formed on the glass. As discussed, this is the consequence of the value of $\theta$. However, for high spectral selectivity between the visible and near-infrared region the coating should be a continuous thin film rather than in the form of discrete islands [26], Fig. 10. This requires coalescence of the gold hemispheres and the creation of a continuous macroscopic network characterized by a 
percolation threshold thickness. At this threshold, sufficient coalescence occurs to create a continuous pathway for electronic conduction through the film, which leads to dramatic changes of conductivity and optical properties. However, the thickness at which this phenomenon occurs is very important in gold coatings for solar control. If it is thicker than $150 \mathrm{~nm}$, the coating will be totally opaque and reflect almost all the radiation in both visible and the near infrared region. Therefore, every effort has to be made to minimize the threshold thickness of nanoscale gold coatings. There are two possible strategies to achieve this. The first is to form the smallest particles possible and ensure that they are copiously nucleated. Growth of such a coating will cause coalescence into a continuous film. The second strategy is to alter the interfacial surface energy, which in its ultimate form will lead the occurrence of the so-called Frank-van der Merwe mode of film formation [27]. In this mode, layers of coatings grow on top of each other, Fig. 11. Naturally, the two strategies can also be combined.

\section{Conclusion}

Glass surfaces were pretreated with 1:1 sulfuric acid $\left(\mathrm{H}_{2} \mathrm{SO}_{4}\right), 98 \% \mathrm{H}_{2} \mathrm{SO}_{4}$ and buffered hydrofluoric acid (HF). The surface obtained with buffered HF had higher sodium content and lower contact angle compared with that treated with $98 \% \mathrm{H}_{2} \mathrm{SO}_{4}$. There was also a marked difference in the morphology and transmission spectra of the gold coatings that were subsequently deposited on the glass. This was evident in the texture of the coating on glass pretreated with HF, which was more uniform than that of the sample pretreated with sulfuric acid, and which exhibited only one absorption peak in its transmission spectrum. These trends could be explained in terms of classic heterogeneous nucleation and growth theory. The optimum microstructure for high 
spectral selectivity has been identified, and is a near-continuous monolayer of gold nanoparticles of less than $\sim 10 \mathrm{~nm}$ diameter.

\section{Acknowledgement}

The authors acknowledge the support of Anglogold Ashanti Australia Ltd.

\section{References}

[1] T.E. Johnson, Low-e glazing Design Guide, Butterworth Architecture, Boston, 1991.

[2] ASTM G159-98 Standard Tables for Solar Spectral Irradiance at Air Mass 1.5, American Society for Testing and Materials, 1998.

[3] J.M. Bell, J.P. Matthews, Materials Forum 1 (1998) 22.

[4] G.B. Smith, C.A. Deller, P.D. Swift, J. Nanoparticle Research 4 (2002) 157.

[5] X. Xu, M. Stevens, M.B. Cortie, Chem. Mater. 16 (2004) 2259.

[6] H. Chowdhury, X. Xu, P. Huynh, M.B. Cortie, J. Solar Energy Eng. 127 (2005) 70 .

[7] S. Chaudhuri, D. Bhattacharyya, A.B. Maity, Mater. Sci. Forum 246 (1997) 181.

[8] H.K. Jang, Y.D. Chung, S.W. Whangbo, J. Vacuum Sci. and Tech. A 18 (2000) 401.

[9] H. Niederprum, H.G. Klein, J. Meussdoerffer, US Patent 4055458, 1977.

[10] S.H. Gimm, J.H. Kim, US Patent 5281350, 1994.

[11] A. Doron, E. Joselevich, A. Schlittner, I. Willner, Thin Solid Films 340 (1999) 183.

[12] B. Kim, L. Tripp, A. Wei, Mater. Res. Soc. Symp. Proc. 676 (2001) Y6.1.1.

[13] L.M. Liz-Marzan, Materials Today Feb. (2004) 26.

[14] T. Nomura, M. Alonso, Y. Kousaka, K.Tanaka, J. Colloid Interf. Sci. 203 (1998) 170.

[15] M.F. Ashby, D.R.H Jones, Engineering Materials 2: An Introduction to Microstructures, Processing and Design, Pergamon Press, Oxford, 1986.

[16] R.C. Weast, Handbook of Chemistry and Physics, $56^{\text {th }}$ ed., CRC Press, Cleveland, Ohio, 1975.

[17] L. Genzel, T.P Martin, Surf. Sci. 34 (1973) 33.

[18] J. Turkevich, Gold Bull. 18 (1985) 125.

[19] M. Quinten J. Cluster Sci. 10 (1999) 319.

[20] T. Ung, L.M. Liz-Marzán, P. Mulvaney, Colloids and Surf. A 202 (2002) 119.

[21] V. Salgueiriño-Maceira, F. Caruso, L. M. Liz-Marzán, J. Phys. Chem. B 107 (2003) 10990.

[22] S.L.Westcott, S.J.Oldenburg, T.R. Lee, N.J.Halas, Chem. Phys. Lett. 300 (1999) 651.

[23] T.J. Norman, J.C.D. Grant, D. Magana, J. Z. Zhang, J. Liu, D. Cao, F. Bridges, A. V. Buuren J. Phys. Chem. B 106 (2002) 7005. 
[24] M. Schmeits, L. Dambly, Phys. Rev. B 44 (1991) 12706.

[25] P. Nordlander, C. Oubret, E.Prodan, K. Li, M.I. Stockman, Nano Lett. 4 (2004) 899.

[26] O.S. Heavens, Thin Film Physics, Methuen \& Co, London, 1970.

[27] N. Kaiser, H.K. Pulker, Optical Interference Coatings, Springer, Berlin, 2003. 
Table 1 Different pre-treatment process for the surface of the glass

\begin{tabular}{llll}
\hline & $\mathrm{A}$ & $\mathrm{B}$ & $\mathrm{C}$ \\
\hline Processing & $1: 1 \mathrm{H}_{2} \mathrm{SO}_{4}$ boiling & $98 \% \mathrm{H}_{2} \mathrm{SO}_{4}$ boiling & Buffered \\
& for 30 minutes[8] & for 30 minutes & hydrofluoric acid, \\
& & & 1 minute \\
\hline
\end{tabular}




\section{List of Figures}

Fig. 1. Hardball model to correct overestimation of radius of gold nanoparticles by atomic force microscopy.

Fig. 2. XPS spectrum for $\mathrm{Na}$ and Si on glass surface with different pretreatments.

Fig. 3. Morphology of glass surfaces produced by different etching processes.

Fig. 4. Transmittance spectrum of gold nano-hemisphere coatings on glass with different pretreatments of the surface.

Fig. 5. Morphology of gold nanoparticles on glass with different pretreatments of the surface.

Fig. 6. Size distribution of gold nanoparticles on glass prepared with different pretreatments of the surface.

Fig. 7. AFM images of gold nanoparticles on glass surfaces with different etching processes.

Fig. 8. Aspect ratio (radius/height) of particles deposited on surface treated with 98\% $\mathrm{H} 2 \mathrm{SO} 4$ compared to that of particles deposited on surface treated with buffered HF. (The trend lines shown are merely to guide the eye.)

Fig. 9. The Gibbs free energy of $10^{-4}$ mol of gold caps compared for two surfaces as a function of particle size.

Fig. 10. Nature of reflection off gold coatings at various stages in the deposition process, redrawn from [20].

Fig. 11. Illustration of the process of nucleation and growth of gold coatings on glass, redrawn from [20]. 